\title{
Generalized Conchoids
}

\section{Generalized Conchoids \\ ABSTRACT}

We adapt the classical definition of conchoids as known from the Euclidean plane to geometries that can be modeled within quadrics. Based on a construction by means of cross ratios, a generalized conchoid transformation is obtained. Basic properties of the generalized conchoid transformation are worked out. At hand of some prominent examples - line geometry and sphere geometry - the actions of these conchoid transformations are studied. Linear and also non-linear transformations are presented and relations to well-known transformations are disclosed.

Key words: conchoid transformation, line geometry, sphere geometry, cross ratio, regulus, Dupin cyclide, Laguerre transformation, equiform transformation, inversion

MSC2010: 14J26, 51C99, 51N05, 51N15, 51N35, 53A05, 53A17, 74N10, 93B17

\section{Introduction}

The well-known construction of conchoids is usually applied to curves in the Euclidean plane $\mathbb{R}^{2}$. Several conchoids of simple and elementary curves in the Euclidean plane are known and have undergone intensive investigations, see for example [4, 6, 10, 11, 16, 22].

The conchoid construction uses a focus $F$ and a directrix $l$ (with $F \notin l$ in case of a straight line $l$ ). Then, a value $d \in \mathbb{R}$ is chosen and the conchoid $c_{d}$ of $l$ with respect to $F$ is defined as

$c_{d}:=\left\{X_{d}: \overline{X_{d} X}=d, X_{d} \in[X, F], \forall X \in l\right\}$

where $\overline{X_{d} X}$ denotes the Euclidean distance of the segment $X_{d} X$ and $[F, X]$ means the line spanned by $F$ and $X$. A special example is obtained by choosing $l$ as a straight line which yields the one-parameter family of Nikomedes's conchoids. Pascal's limaçon is the conchoid of a circle $l$ with $F \in l$, see $[6,11,22]$. Fig. 1 shows some members from the Nikomedes family. In Euclidean geometry

\section{Poopćene konhoide SAŽETAK}

Prilagođavamo klasičnu definiciju konhoida iz euklidske ravnine geometrijama definiranim kvadrikama. Postiže se poopćena konhoidna transformacija koja se temelji na konstrukciji pomoću dvoomjera. Proučavaju se osnovna svojstva ovakve transformacije. Djelovanje poopćene konhoidne transformacije se proučava na nekim istaknutim primjerima kao što su pravčasta i sferna geometrija. Prikazuju se linearne i nelinearne transformacije te su opisane veze $\mathrm{s}$ dobro poznatim transformacijama.

Ključne riječi: konhoidna transformacija, pravčasta geometrija, sferna geometrija, dvoomjer, sustav izvodnica, Dupinova ciklida, Laguerrova transformacija, ekviformna transformacija, inverzija

it makes a difference whether the distance $d$ is equipped with a sign or not. So, the conchoid $c_{d}$ has either one or two branches depending on whether $d$ is signed or not.

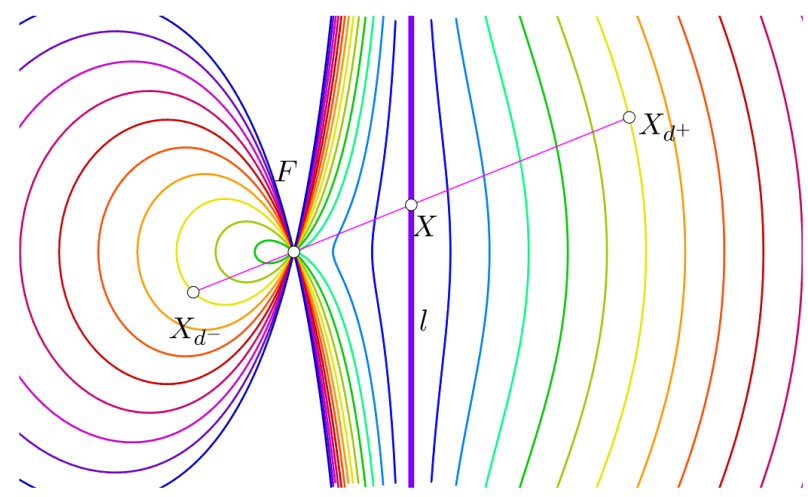

Figure 1: Some conchoids of a straight line l with respect to the focus $F$. 
In the following, the mapping $X \mapsto X_{d}$ shall be called the conchoid transformation. It is clearly seen that the conchoid transformation defined via (1) can be applied to arbitrary submanifolds of any metric space. Thus, the conchoid transformation has been applied to surfaces in Euclidean three-space $\mathbb{R}^{3}$ in $[13,14,15,17,18]$ in order to construct new classes of surfaces admitting rational parametrizations, and thus, making them accessible to the algorithms implemented in CAD systems. In [8], a special affine version of a line geometric conchoid transformation was presented. Conchoids on the Euclidean unit sphere were studied from the algebraic and constructive point of view in [12].

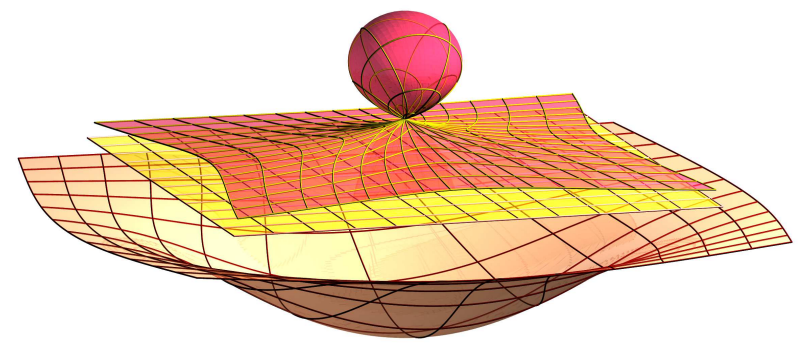

Figure 2: The conchoid of a plane with respect to a point is a surface of revolution with Nikomedes's conchoid for its meridian curve.

None of the constructions presented in $[13,14,15]$ preserves the type of the geometric object that undergoes the conchoid transformation. The conchoids of planes and spheres become some algebraic surfaces that somehow imitate the features of the conchoids known from the Euclidean plane, see Fig. 2. Ruled surfaces transform to arbitrary surfaces which, in general, carry maybe only a finite number of straight lines, cf. [13].

In this paper, we adapt the conchoid construction such that it applies to various geometries that can be modeled within quadrics. This is especially the case for the geometry of lines and spheres in three-dimensional spaces which can be modeled within Plücker's and Lie's quadric. So, we are able to find conchoids within certain classes of geometric objects: Lines or spheres can be mapped to lines and spheres. Consequently, ruled surfaces and channel surfaces are transformed to such surfaces. As a by product, rational parametrizations are also preserved.

However, this concept is not restricted to line and sphere geometry, but these are taken as examples in order to show how the generalized conchoid transformation acts. The group of Euclidean motions can be treated in Study's quadric model and also Möbius geometry can be realized on a sphere, further, isotropic geometries and Laguerre geometry also have quadric models and the generalized conchoid transformation can be used there. We shall not discuss these latter four in detail.
Section 2 is dedicated to the generalized conchoid construction and its basic properties. The special cases of line and sphere geometric conchoid transformations are discussed in Sections 3 and 4. In both sections, we treat linear conchoid transformations and special types of quadratic transformations.

\section{The generalized conchoid transformation}

Let $\mathbb{F}$ be an arbitrary commutative field with char $\mathbb{F} \neq 2$. Further, let $\mathbb{F}^{n+1}$ be the $(n+1)$-dimensional vector space on $\mathbb{F}$ and $\mathbb{P}^{n}(\mathbb{F})$ be the projective space of $n$ dimensions over $\mathbb{F}^{n+1}$. A quadric $Q \subset \mathbb{P}^{n}(\mathbb{F})$ can be defined by prescribing a symmetric bilinear form $\Omega: \mathbb{F}^{n+1} \times \mathbb{F}^{n+1} \rightarrow \mathbb{F}$. With $\mathbf{x}$ we denote the homogeneous coordinate vector of a point $X \in \mathbb{P}^{n}(\mathbb{F})$ and the equation of the quadric $Q$ is then $\Omega(\mathbf{x}, \mathbf{x})=0$. In the following, lower case bold letters denote the coordinate vectors while capitals denote the points, i.e., the point $P$ has the coordinate vector $\mathbf{p}$.

Assume that we are given three points $P_{i}(i \in\{0,1,2\})$ in a quadric $Q \in \mathbb{P}^{n}(\mathbb{F})$. The line $\left[P_{0}, P_{1}\right]$ shall not be contained in the quadric and the plane $\pi=\left[P_{0}, P_{1}, P_{2}\right]$ shall not be tangent to $Q$. Further, $f:=\pi \cap Q$ shall be a regular conic in $Q$. Then, there is always a uniquely defined point $P_{\delta}$ that forms the cross ratio $\delta=\operatorname{cr}\left(P_{0}, P_{1}, P_{2}, P_{\delta}\right)$ with $P_{0}, P_{1}$, and $P_{2}$. Now, we give

Definition 1 To any triple $\left(P_{0}, P_{1}, P_{2}\right)$ of three different points in a quadric $Q \subset \mathbb{P}^{n}(\mathbb{F})$ and to any value $\delta \in$ $\mathbb{F} \cup\{\infty\}$ there exists a uniquely defined fourth point $P_{\delta}$ such that $\operatorname{cr}\left(P_{0}, P_{1}, P_{2}, P_{\delta}\right)=\delta$ provided that $\left[P_{0}, P_{1}\right] \not \subset Q$ $\pi=\left[P_{0}, P_{1}, P_{2}\right]$ is not tangent to $Q$ and char $\mathbb{F} \neq 2$.

We call $P_{\delta}$ the $\delta$-conchoid transformation of $P_{2}$ with respect to the foci $P_{0}$ and $P_{1}$.

Later, we will apply the thus generalized conchoid construction to points in ruled quadrics. Therefore, we do not exclude the case of collinear points $P_{0}, P_{1}$, and $P_{2}$. Moreover, four points in a quadric do not have to be coplanar in order to assign a cross ratio to them.

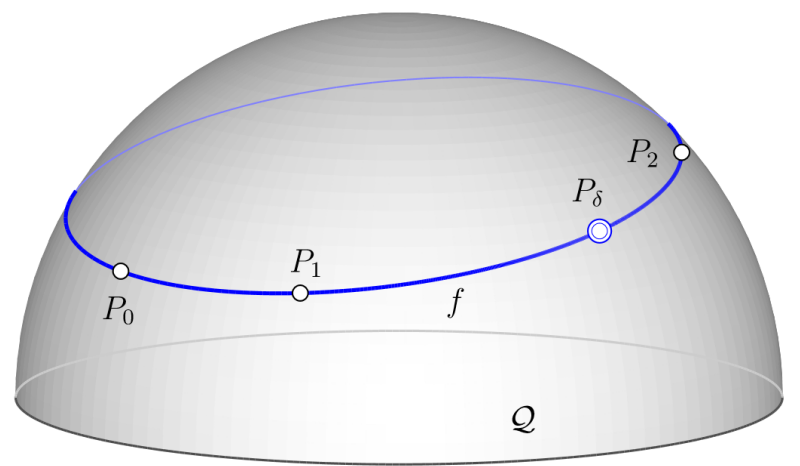

Figure 3: The generalized conchoid transformation acting on a quadric. 
By means of a stereographic projection to the Gauss plane, we obtain four points that can be identified with four complex numbers and the definition of a cross ratio of these four points is straight forward, see [2].

Remark 1 1. None of the three points $P_{0}, P_{1}, P_{2}$ is distinguished. In fact, any two points $P_{i}$ and $P_{j}(i \neq j)$ out of the three initial points can be considered the foci of the conchoid transformation. Then, $P_{\delta}$ is the conchoid transform of $P_{k}(k \neq i, j)$ with respect to $P_{i}$ and $P_{j}$.

2. Changing the roles of the given points does not really change the conchoid transformation. Only the cross ratio $\delta$ may turn into one of the six values $\delta$, $\delta^{-1}, 1-\delta,(1-\delta)^{-1}, \delta(\delta-1)^{-1},(\delta-1) \delta^{-1}$.

3. In the case char $\mathbb{F}=3$, only the cross ratio $\delta=2$ yields a conchoid transform $P_{\delta} \neq P_{0}, P_{1}, P_{2}$. Since any projective line, and thus, any projective conic carries four points in this case, the conchoid transform $P_{\delta}$ of any three (collinear or con-conic) points is the remaining fourth point. Furthermore, 2 is its additive inverse, $P_{\delta}$ makes $P_{0}, P_{1}, P_{2}$ a harmonic quadruple. Therefore, the conchoid transform $P_{\delta}$ of $P_{i}$ with respect to $P_{j}$ and $P_{k}$ (with $i \neq j, k$ and $j \neq k$ ) is the harmonic conjugate of $P_{i}$ with respect to $P_{j}$ and $P_{k}$.

We can give a coordinate representation of the generalized conchoid transformation in $\mathbb{P}^{n}(\mathbb{F})$ which is helpful for further investigations:

Theorem 1 Let $\mathbb{F}^{n+1}$ be the $(n+1)$-dimensional vector space on the commutative field $\mathbb{F}$ with char $\mathbb{F} \neq 2$. Assume that $\Omega: \mathbb{F}^{n+1} \times \mathbb{F}^{n+1} \rightarrow \mathbb{F}^{n+1}$ is a symmetric nondegenerate bilinear form defining a quadric $Q \subset \mathbb{P}^{n}(\mathbb{F})$ by $Q: \Omega(\mathbf{x}, \mathbf{x})=0$.

Then, the conchoid transformation $P_{2} \mapsto P_{\delta}$ as explained in Def. 1 can be given in terms of homogeneous point coordinates in $\mathbb{P}^{n}(\mathbb{F})$ as

$\mathbf{p}_{\delta}=\delta(\delta-1) \Omega_{12} \mathbf{p}_{0}+(1-\delta) \Omega_{02} \mathbf{p}_{1}+\delta \Omega_{01} \mathbf{p}_{2}$

where $\delta \in \mathbb{F} \cup\{\infty\}, \mathbf{p}_{i}(i \in\{0,1,2\})$ are the homogeneous coordinates of the points $P_{i}, \Omega_{i j}:=\Omega\left(\mathbf{p}_{i}, \mathbf{p}_{j}\right)$, and neither pair $\left(\mathbf{p}_{i}, \mathbf{p}_{j}\right)$ is conjugate with regard to $Q$ (i.e., $\left.\Omega_{i j} \neq 0\right)$.

Proof. We prove Thm. 1 by constructing the coordinate representation given in (2). In the end, we shall arrive at a parametrization of the conic $f=\left[P_{0}, P_{1}, P_{2}\right] \cap Q$ with

$\operatorname{cr}\left(P_{0}, P_{1}, P_{2}, P_{\delta}\right)=\delta$.

For that, we observe

$\operatorname{cr}\left(P_{0}, P_{1}, P_{2}, P_{\delta}\right)=\operatorname{cr}\left(T_{P_{0}},\left[P_{0}, P_{1}\right],\left[P_{0}, P_{2}\right],\left[P_{0}, P_{\delta}\right]\right)=\delta$ where $T_{P_{0}}$ is the tangent of $f$ at $P_{0}$ (see Fig. 4), i.e., the intersection of the tangent hyperplane $T_{P_{0}} Q$ of $Q$ at $P_{0}$ with the plane $\left[P_{0}, P_{1}, P_{2}\right]$. The lines $T_{P_{0}},\left[P_{0}, P_{1}\right],\left[P_{0}, P_{2}\right],\left[P_{0}, P_{\delta}\right]$ from the pencil about $P_{0}$ establish the stereographic projection $f \rightarrow\left[P_{1}, P_{2}\right]$ which preserves cross ratios. If further $T_{0}:=T_{P_{0}} \cap\left[P_{1}, P_{2}\right]$ and $P_{\delta}^{\prime}:=\left[P_{0}, P_{\delta}\right] \cap\left[P_{1}, P_{2}\right]$, then $\operatorname{cr}\left(P_{0}, P_{1}, P_{2}, P_{\delta}\right)=\operatorname{cr}\left(T_{0}, P_{1}, P_{2}, P_{\delta}^{\prime}\right)=\delta$.

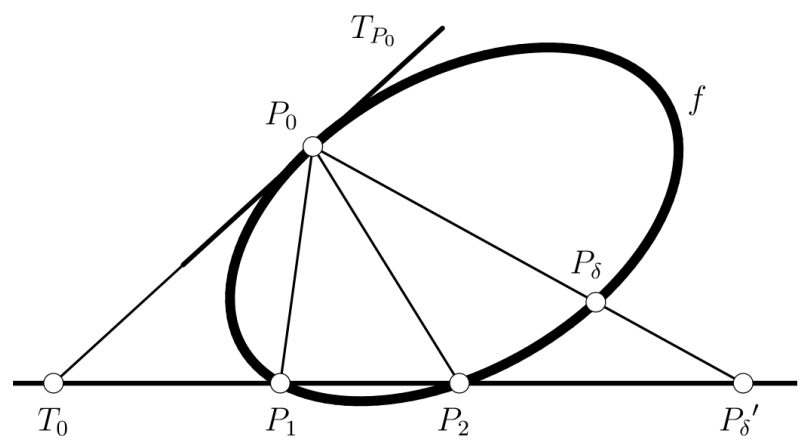

Figure 4: The stereographic projection $f \rightarrow\left[P_{1}, P_{2}\right]$ yields a parametrization of the conic $f$ by means of the cross ratio $\delta$.

The point $T_{0}$ is found as the common point of $T_{P_{0}} Q$ : $\Omega\left(\mathbf{p}_{0}, \mathbf{x}\right)=0$ and the line $\mathbf{g}_{12}(\lambda, \mu)=\lambda \mathbf{p}_{1}+\mu \mathbf{p}_{2}$ (with $\left.(\lambda, \mu) \in \mathbb{F}^{2} \backslash\{(0,0)\}\right)$. This yields

$\mathbf{t}_{0}=-\Omega_{02} \mathbf{p}_{1}+\Omega_{01} \mathbf{p}_{2}$

Obviously, $\mathbf{t}_{0} \neq \mathbf{p}_{i}$ for $\Omega_{0 i} \neq 0$ for $i \in\{1,2\}$, by assumption. Now, the pairs $\left(-\Omega_{02}, \Omega_{01}\right),(1,0),(0,1)$ are homogeneous coordinates of $T_{0}, P_{1}, P_{2}$ on the line $\left[P_{1}, P_{2}\right]$, and thus, we find $P_{\delta}^{\prime}$ with $\operatorname{cr}\left(T_{0}, P_{1}, P_{2}, P_{\delta}^{\prime}\right)=\delta$ as

$\mathbf{p}_{\delta}^{\prime}=(1-\delta) \Omega_{02} \mathbf{p}_{1}+\delta \Omega_{01} \mathbf{p}_{2}$.

The stereographic projection $\left[P_{1}, P_{2}\right] \rightarrow Q$, and thus, also onto $f$ with center $P_{0}$ sends $P_{\delta}^{\prime}$ to $P_{\delta}$. Since $P_{\delta}=Q \cap$ $\left[P_{0}, P_{\delta}^{\prime}\right] \backslash\left\{P_{0}\right\}$, we find $\mathbf{p}_{\delta}=\mathbf{p}_{\delta}^{\prime}+\delta(\delta-1) \Omega_{12} \mathbf{p}_{0}$ which completes the proof.

The coordinate representation

$\mathbf{p}_{\delta}: \mathbb{F} \cup\{\infty\} \rightarrow Q$

of $P_{\delta}$ given in (2) is a parametrization of the conic $f \subset Q$. Hence, $\Omega\left(\mathbf{p}_{\delta}, \mathbf{p}_{\delta}\right)=0 \forall \delta \in \mathbb{F} \cup\{\infty\}$. We shall call $f$ the fiber (conic) of the conchoid transformation.

From the definition of the generalized conchoid transformation and the analytic representation (2), we can easily conclude:

Corollary 1 The generalized conchoid transformation is involutive if, and only if, the cross ratio equals $\delta=-1$. 
Proof. Geometrically speaking: If $P_{\delta}$ is the conchoid transform of $P_{2}$ with $\delta=-1$ and, if transformed with $\delta=-1$ once again, then we obtain $P_{2}$ back.

Remark 2 If $\operatorname{char} \mathbb{F}=3$, then the non-trivial $(\delta \neq 0,1, \infty)$ generalized conchoid transformation is always involutive.

None of the points $\mathbf{p}_{i}$ is geometrically distinguished which is expressed in (2) by the fact that the coordinate representation of $\mathbf{p}_{\delta}$ is a trilinear form: It is linear in each $\mathbf{p}_{i}$. This gives rise to the following:

Corollary 2 1. The generalized conchoid transformation on a quadric $Q$ can be extended to an automorphic collineation $\kappa$ of $Q$.

2. The collineation $\kappa$ has the fixed points $P_{0}$ and $P_{1}$ corresponding to the two eigenvalues $\delta^{2} \Omega_{01}$ and $\Omega_{01}$.

3. The polar space $F$ of $f:=\left[P_{0}, P_{1}\right]$ with regard to $Q$ is fixed point wise and corresponds to the eigenvalue $\delta \Omega_{01}$.

\section{Proof.}

1. Consider $\mathbf{p}_{2}$ in (2) as the variable point $\mathbf{x}$. Then, we observe that each summand depends only linearly on $\mathbf{x}$ : The first two coefficients in the linear combination are $\Omega\left(\mathbf{p}_{1}, \mathbf{x}\right)$ and $\Omega\left(\mathbf{p}_{0}, \mathbf{x}\right)$ and neither is multiplied with $\mathbf{x}$. Also the last summand depends only linearly on $\mathbf{x}$, since $\Omega_{01}$ is independent of $\mathbf{x}$. Since $\mathbf{p}_{2}=\mathbf{x} \mapsto \mathbf{p}_{\delta}$ is homogeneous and linear in $\mathbf{x}$ and $\mathbf{p}_{\delta} \in Q(\forall \delta \in \mathbb{F} \cup\{\infty\})$, it is an automorphic collineation of $Q$.

2. By letting either $\mathbf{p}_{2}=\mathbf{p}_{0}$ or $\mathbf{p}_{2}=\mathbf{p}_{1}$ in (2), we see that the conchoid transformation returns either $\mathbf{p}_{\delta}=\delta^{2} \Omega_{01} \mathbf{p}_{0}$ or $\mathbf{p}_{\delta}=\Omega_{01} \mathbf{p}_{1}$.

3. The three-dimensional polar space $F$ of $f=\left[P_{0}, P_{1}\right]$ with regard to the quadric $Q: \Omega(\mathbf{x}, \mathbf{x})=0$ is given by the homogeneous linear equations

$$
F: \Omega\left(\mathbf{p}_{0}, \mathbf{x}\right)=\Omega\left(\mathbf{p}_{1}, \mathbf{x}\right)=0 .
$$

With (2), $\mathbf{x} \mapsto \mathbf{x}_{\delta}$ and reads

$\mathbf{x}_{\delta}=\delta(\delta-1) \Omega_{1 \mathbf{x}} \mathbf{p}_{0}+(1-\delta) \Omega_{0 \mathbf{x}} \mathbf{p}_{1}+\delta \Omega_{01} \mathbf{x}$

with $\Omega_{i \mathbf{x}}:=\Omega\left(\mathbf{p}_{i}, \mathbf{x}\right)$ for $i=0,1$. Inserting (3) into (4), we infer $\mathbf{x}_{\delta}=\delta \Omega_{01} \mathbf{x}$ which holds true for all $\mathbf{x} \in \mathbb{F}^{n+1} \backslash\{\mathbf{o}\}$ subject to (3).

Remark 3 The cross ratio itself is a homogeneous coordinate on a projective line or on a conic section (in fact on any rational normal curve). Therefore, we could replace the inhomogeneous parameter $\delta$ in (2) by the homogeneous parameter $\left(d_{0}, d_{1}\right) \in \mathbb{F}^{2} \backslash\{(0,0)\}$ with $\delta=d_{1} d_{0}^{-1}$. We omit this since $d_{0}=0$ causes $\delta=\infty, P_{\delta}=P_{0}$ which shall be excluded from our considerations.

\section{Line geometric conchoids}

\subsection{Linear transformations}

In this section, we apply the generalized conchoid transformation to the manifold of lines. For that we use the well-known Klein model for the set of lines in a threedimensional space. Details, exact definitions, properties, and how to compute with Plücker coordinates can found in $[9,19,20,23]$.

We describe lines $L$ in projective three-space $\mathbb{P}^{3}(\mathbb{F})$ by Plücker coordinates

$(\mathbf{l} ; \overline{\mathbf{l}})=\left(l_{1}, l_{2}, l_{3} ; l_{4}, l_{5}, l_{6}\right) \in \mathbb{F}^{6} \backslash\{\mathbf{o}\}$

which can be made homogeneous (see [9, 19, 20, 23]). Thus, they can be interpreted as coordinates of points in a projective space of five dimensions. From the definition of Plücker coordinates of lines in three-space it is clear that these six-tuples satisfy a quadratic relation:

$M_{2}^{4}: \frac{1}{2} \Omega_{L}(L, L)=\langle\mathbf{l}, \overline{\mathbf{l}}\rangle=l_{1} l_{4}+l_{2} l_{5}+l_{3} l_{6}=0$.

$M_{2}^{4}$ is a quadric of four dimensions. It is of index two, i.e., the maximum dimension of subspaces contained in $M_{2}^{4}$ equals two. All points on $M_{2}^{4}$ correspond to lines in $\mathbb{P}^{3}(\mathbb{F})$ and any line can be described by Plücker coordinates satisfying (6). (An affine or a Euclidean specialization is also possible, see [20, 23].)

The polar form $\Omega_{L}: \mathbb{F}^{6} \times \mathbb{F}^{6} \rightarrow \mathbb{F}$ of $M_{2}^{4}$ can be used to characterize pairs of lines. Two different lines $L$ and $M$ are coplanar if, and only if, $\Omega_{L}(L, M)=0$, i.e., the corresponding points in $\mathbb{P}^{5}$ are conjugate with regard to $M_{2}^{4}$.

Since $M_{2}^{4}$ is a regular hyperquadric in $\mathbb{P}^{5}(\mathbb{F})$, there is a nine-parameter family of conics in it. We have to distinguish between three types of regular conics in Plücker's quadric: (1) the transversal intersection of a twodimensional subspace $\mathbb{P}^{2}(\mathbb{F}) \subset \mathbb{P}^{5}(\mathbb{F})$; (2a) a conic in a plane $\mathbb{P}_{1}^{2}(\mathbb{F}) \subset M_{2}^{4}$ (of the first kind); (2b) a conic in a plane $\mathbb{P}_{2}^{2}(\mathbb{F}) \subset M_{2}^{4}$ (of the second kind). In the following, the conic of type (1) is the most important. The points on a conic of that type correspond to one particular oneparameter family of lines in a ruled quadric, i.e., a regulus. The points on the conics mentioned in the cases (2a) and (2b) correspond to the rulings of a quadratic cone or to the tangents of a (planar) conic, see Fig. 5. Consequently, the fibers of the line geometric conchoid transformation are reguli and in some cases the rulings of quadratic cones or the tangents of conics. Fig. 6 shows a typical fiber regulus. 

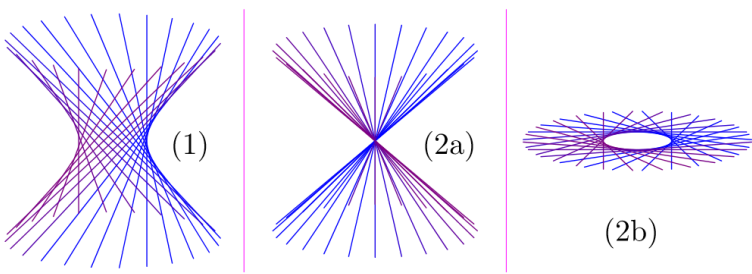

$(2 \mathrm{~b})$

Figure 5: The three types of reguli.

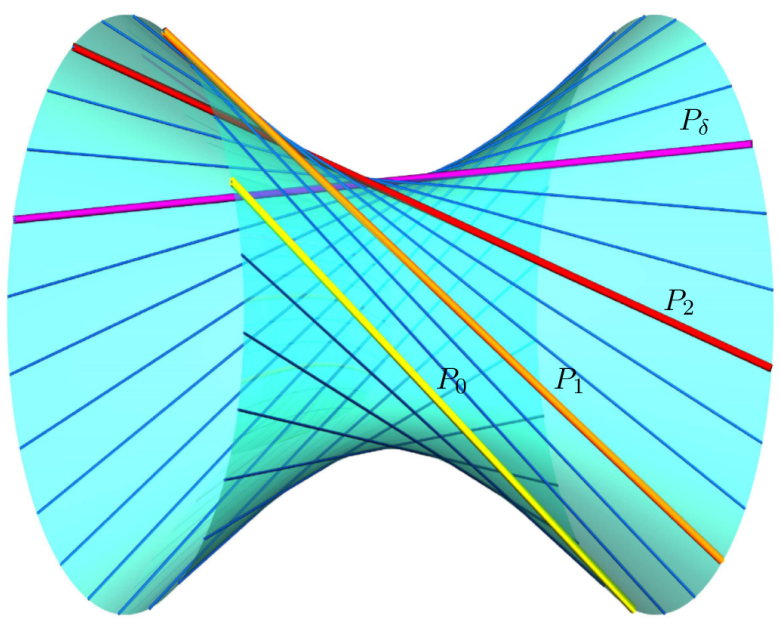

Figure 6: A regular fiber regulus of the line geometric conchoid transformation.

Since the line geometric conchoid transformation maps points on Plücker's quadric to points on Plücker's quadric, it preserves lines, and thus, it maps ruled surfaces to ruled surfaces, and even congruences and complexes of lines (two- and three-dimensional submanifolds of $M_{2}^{4}$ ) to such. By assumption, the case of intersecting focal lines $L_{0}$ and $L_{1}$ is excluded and so we have:

Theorem 2 Let $L_{0}$ and $L_{1}$ be two (skew) lines in $\mathbb{P}^{3}(\mathbb{F})$ and let $\delta \in \mathbb{F} \cup\{\infty\}$ be a certain fixed value. Then, the line geometric conchoid transformation induces a (regular) automorphic collineation $\mathrm{\kappa}$ of $M_{2}^{4}$ that has a fixed line $f \subset \mathbb{P}^{5}(\mathbb{F})$ and a fixed three-space $F \subset \mathbb{P}^{5}(\mathbb{F})$ for its axis. $F$ and $f$ are polar with regard to $M_{2}^{4}$.

Proof. It is always possible to choose homogeneous coordinates in $P^{3}(\mathbb{F})$ such that the Plücker coordinates of the focal lines are

$L_{0}=(1,0,0 ; 0,0,0), L_{1}=(0,0,0 ; 1,0,0)$,

(satisfying $\Omega_{L}\left(L_{0}, L_{1}\right) \neq 0$ ) and $L_{2}=\left(l_{1}, l_{2}, l_{3} ; l_{4}, l_{5}, l_{6}\right)$. We insert into (2) and arrive at

$L_{\delta}=\left(\delta^{2} l_{1}, \delta l_{2}, \delta l_{3} ; l_{4}, \delta l_{5}, \delta l_{6}\right)$.
Obviously, the mapping $\kappa: L_{2} \mapsto L_{\delta}$ is a regular collineation with the diagonal matrix $D=$ $\operatorname{diag}\left(\delta^{2}, \delta, \delta, 1, \delta, \delta\right)$ for its coordinate representation. The linear mapping described by $D$ fixes the quadratic form $\Omega_{L}$, and thus, it is an automorphic collineation of $M_{2}^{4}$. The eigenvalues are $t_{1}=1, t_{2}=\delta^{2}$, and $t_{3}=\delta$ with their algebraic multiplicities $\mu\left(t_{1}\right)=\mu\left(t_{2}\right)=1$ and $\mu\left(t_{3}\right)=4$. The fixed points corresponding to 1 and $\delta^{2}$ are $L_{0}$ and $L_{1}$, while $\delta$ determines the $\kappa$-invariant three-space $F: x_{1}=x_{4}=0$. The restriction $\left.\kappa\right|_{f}$ of $\kappa$ to the line $f:=\left[L_{0}, L_{1}\right]$ is a hyperbolic projective mapping with two fixed points $L_{0}, L_{1}$ and the coordinate representation $\operatorname{diag}\left(\delta^{2}, 1\right)$. The three-space $F$ is fixed pointwise under $\kappa$ and is polar to $f$ according to Cor. 2.

Remark 4 In the case of intersecting focal lines $L_{0}$ and $L_{1}$, i.e., $\Omega_{L}\left(L_{0}, L_{1}\right)=0, \kappa$ is a projection (singular collineation) onto a one-dimensional subspace of $\mathbb{P}^{5}(\mathbb{F})$ as can be seen by choosing

$L_{0}=(1,0,0 ; 0,0,0), L_{1}=(0,1,0 ; 0,0,0)$

and $L_{2}=\left(l_{1}, l_{2}, l_{3} ; l_{4}, l_{5}, l_{6}\right)$. With (2), we find $L_{\delta}=$ $\left(-\delta l_{5}, l_{4}, 0 ; 0,0,0\right)$ provided that $\delta \neq 0$ otherwise $L_{\delta}$ is a single point. Now, $L_{2} \mapsto L_{\delta}$ is a projection onto the subspace $x_{2}=x_{3}=x_{5}=x_{6}=0$. Note that $f$ and $F$ are polar with regard to $M_{2}^{4}$.

We take closer look at the induced collineation of the regular (linear) line geometric conchoid transformation. The incidence graph in Fig. 7 shall support our imagination.

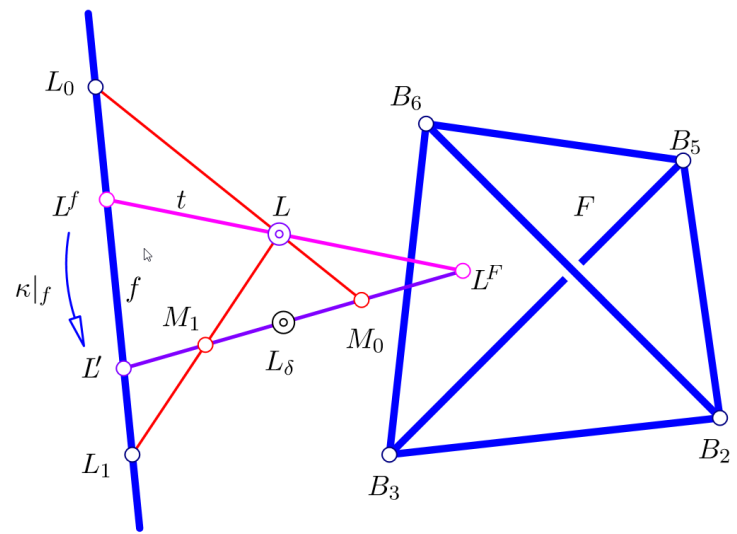

Figure 7: Action of the collineation induced by the regular (linear) line geometric conchoid transformation.

Through a generic point $L \in \mathbb{P}^{5}(\mathbb{F})$ there exists a unique line $t$ that meets $f$ and $F$. We denote the intersections of $t$ and the fixed spaces $f, F$ by $L^{f}$ and $L^{F}$. The projective mapping $\left.\kappa\right|_{f}: f \rightarrow f$ sends $L^{f}$ to a point $L^{\prime}$ while $\left.\kappa\right|_{F}=\mathrm{id}_{F}$ and $L^{F}$ remains fixed. Thus, $\kappa$ maps $t$ to the line $t^{\prime}=\left[L^{\prime}, L^{F}\right]$. Since $\kappa$ preserves collinearities, the image $L_{\delta}$ 
of $L$ has to be on $t^{\prime}$. The projections $M_{0}$ and $M_{1}$ of $L$ from $L_{0}$ and $L_{1}$ onto $t^{\prime}$ satisfy

$\operatorname{cr}\left(L^{\prime}, L^{F}, M_{0}, L_{\delta}\right)=\delta^{-1}, \quad \operatorname{cr}\left(L^{\prime}, L^{F}, M_{1}, L_{\delta}\right)=\delta$.

We are able to show that the automorphic collineations of $M_{2}^{4}$ obtained from the line geometric conchoid transformation are indeed induced by projective collineations $\mathbb{P}^{3}(\mathbb{F}) \rightarrow \mathbb{P}^{3}(\mathbb{F}):$

Theorem 3 Any automorphic collineation $\kappa$ of $M_{2}^{4}$ induced by the line geometric conchoid transformation is induced by a projective collineation $\alpha: \mathbb{P}^{3}(\mathbb{F}) \rightarrow \mathbb{P}^{3}(\mathbb{F})$.

Proof. It means no restriction to assume that the focal lines $L_{0}$ and $L_{1}$ are those used in the proof of Thm. 2. (The coordinate system in $\mathbb{P}^{3}(\mathbb{F})$ can always be chosen appropriately.) Then, according to the proof of Thm. 2, the automorphic collineation $\kappa$ of $M_{2}^{4}$ induced by the line geometric conchoid transformation is given by (7) and is described by the diagonal matrix $D:=\operatorname{diag}\left(\delta^{2}, \delta, \delta, 1, \delta, \delta\right)$. Now, we have to show that $D$ can be written as the Kronecker product $A \otimes A$ of a (regular) $4 \times 4$ matrix $A$ with itself being a the transformation matrix of a collineation $\alpha: \mathbb{P}^{3}(\mathbb{F}) \rightarrow \mathbb{P}^{3}(\mathbb{F})$. It turns out that $A=\operatorname{diag}(\delta, \delta, 1,1)$ fulfills the equation $D=A \oplus A$, and thus, the linear mapping given by $D$ describing an automorphic collineation of $M_{2}^{4}$ is really induced by a projective collineation $\alpha: \mathbb{P}^{3}(\mathbb{F}) \rightarrow \mathbb{P}^{3}(\mathbb{F})$ with coordinate matrix $A$.

The factorization of the $6 \times 6$ matrix $D$ given in the proof of Thm. 3 may not be unique. However, the uniqueness is not necessary in order to show that the collineation $\kappa: M_{2}^{4} \rightarrow M_{2}^{4}$ described by $D$ is induced by a collineation $\alpha: \mathbb{P}^{3}(\mathbb{F}) \rightarrow \mathbb{P}^{3}(\mathbb{F})$ as long as there exists at least one.

Assume now that $\mathbb{F}=\mathbb{R}$ and $\mathcal{R}: I \subset \mathbb{R} \rightarrow M_{2}^{4}$ is a curve in $M_{2}^{4}$. Then, it corresponds to a ruled surface in $\mathbb{P}^{3}(\mathbb{R})$. A regular point $R=\mathcal{R}\left(t_{0}\right)$ on this curve corresponds to a regular ruling on the ruled surface in $\mathbb{P}^{3}(\mathbb{R})$. The regular ruling $R$ is called torsal if $\dot{R}=\dot{R}\left(t_{0}\right)$ fulfills $\Omega_{L}(\dot{R}, \dot{R})=0$. Along a torsal ruling the tangent planes of the ruled surface do not change, see $[9,19]$.

A ruled surface that consists of torsal rulings only is called torsal ruled surface and its parametrization $\mathcal{R}(t)$ satisfies $\Omega_{L}(\dot{\mathcal{R}}, \dot{\mathcal{R}})=0$ besides $\Omega_{L}(\mathcal{R}, \mathcal{R})=0$, both for all $t \in I$.

The term torsal ruled surface covers cylinders, cones, and the surfaces swept by the tangents of a (space) curve (in $\mathbb{P}^{3}(\mathbb{R})$ ). Torsal ruled surfaces in Euclidean three-space can be mapped isometrically onto a Euclidean plane, and therefore, these surfaces are called developable. However, torsality is a projective differential geometric property of a ruled surface (see $[9,19,20,23])$ and we can say:

Corollary 3 Torsal ruled surfaces are mapped to torsal ruled surfaces under the linear line geometric conchoid transformation.
Proof. Since torsality of rulings and ruled surfaces is a projective property, it cannot be harmed by the induced automorphic collineation of $M_{2}^{4}$. According to Thm. 3, the latter is induced by a projective collineation in $\mathbb{P}^{3}$.

We could also prove the corollary by direct calculation. Assume that $\mathcal{R}: I \subset \mathbb{R} \rightarrow M_{2}^{4}$ is a curve in $M_{2}^{4}$ (i.e., $\Omega_{L}(\mathcal{R}, \mathcal{R})=0 \forall t \in I$ ) all of whose rulings are torsal, i.e., $\Omega_{L}(\dot{\mathcal{R}}, \dot{\mathcal{R}})=0$ for all $t \in I$. Then, we compute $\mathcal{R}_{\delta}$ with (2), differentiate once with respect to $t$, and verify that $\Omega_{L}\left(\dot{\mathcal{R}}_{\delta}, \dot{\mathcal{R}}_{\delta}\right)=0$ on $I \subset \mathbb{R}$.

Some examples shall illustrate the action of the (linear) line geometric conchoid transformation:

Example 1 The tangents of the curve $\left(6,6 t, 3 t^{2}, 2 t^{3}\right) \subset$ $\mathbb{P}^{3}(\mathbb{R})$ sweep a cubic developable. If we choose $L_{0}=$ $(1,0,0 ; 0,0,0), L_{1}=(0,0,0 ; 1,0,0)$ and insert into (2), we obtain the cubic developable built by the tangents of the cubic $\left(6 \delta, 6 \delta t, 3 t^{2}, 2 t^{3}\right)$. If we slice $\mathbb{P}^{3}(\mathbb{F})$ along $x_{0}=0$ (as usual), we see that the two cubics are related by an affine transformation and so are the cubic developables. Fig. 8 shows the initial cubic developable together with three of its line geometric conchoids. We can see that the parametrization of the two cubic curves differ only by a multiplication with the matrix $A=\operatorname{diag}(\delta, \delta, 1,1)$ given in the proof of Thm. 3.

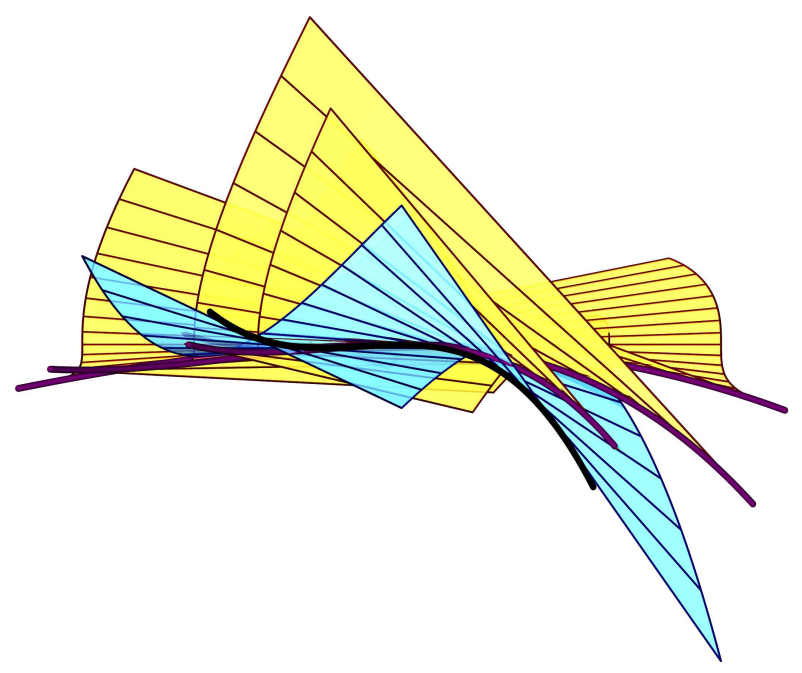

Figure 8: Some cubic developables being each others line geometric conchoids.

Example 2 Assume that the focal lines are $L_{0}=$ $(0,-1,2 ; 0,-2,-1)$ and $L_{1}=(1,1,3 ; 3,0,-1)$. Further, we choose $\delta=2 / 5$ and apply the line geometric conchoid transformation (2) to the set of rulings given by $L_{2}(t)=\left(t^{2}+t, t-t^{2}, 2,-t-t^{2}, t^{2}-t, t^{4}+t^{2}\right)$ and $o b-$ tain $L_{d}(t)=\left(30 t^{4}-25 t^{2}-85 t-30,48 t^{4}+151 t^{2}-79 t-\right.$ $42,54 t^{4}+63 t^{2}-57 t-206,90 t^{4}+205 t^{2}+25 t-90,36 t^{4}+$ $\left.2 t^{2}+82 t-24,-82 t^{4}-79 t^{2}+21 t+18\right)$. A part of this 
ruled surface is shown in Fig. 9 together with the focal lines and one fiber regulus.

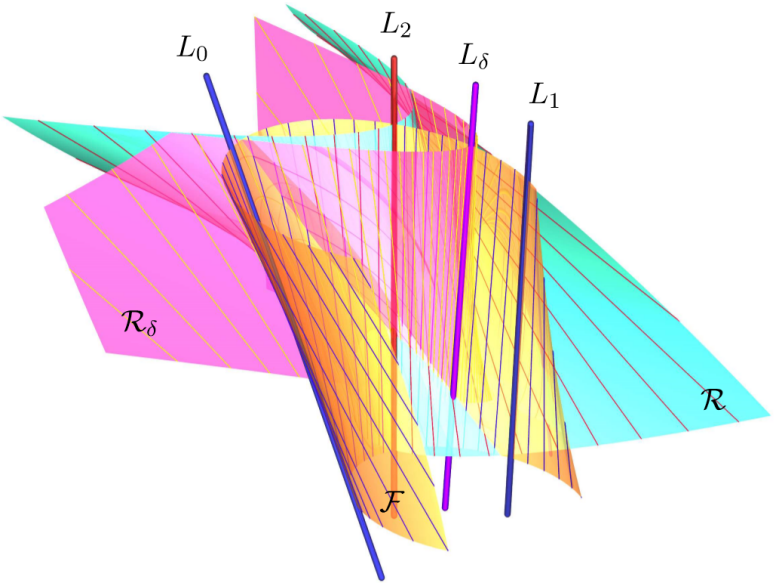

Figure 9: The conchoid transform of a quartic ruled surface with the two focal lines $L_{0}, L_{1}$, and a fiber regulus $\mathcal{F}$.

\subsection{Quadratic mappings}

The line geometric conchoid transformations discussed in the previous section turned out to be linear mappings, i.e., collineations. From that it is a small step to the definition of a quadratic mapping: Assume that one focal line, say $L_{1}$, is image of $L_{2}$ under a fixed projective transformation and leave $L_{0}$ fixed. Then, (2) yields a transformation in terms of Plücker coordinates $\left(l_{1}, \ldots, l_{6}\right)$ that is quadratic in the $l_{i}$. A special affine version of such a quadratic line geometric conchoid transformation was studied in [8].

In the following, we shall consider a special Euclidean version of a quadratic line geometric conchoid transformation. For that purpose, we assume that $L_{0}=(1,0,0 ; 0,0,0)$ is the fixed and constant first focal line. It means no restriction to assume that $L_{0}$ coincides with the $x$-axis of the coordinate system. The second focal line shall be the absolute polar of the line $L_{2}=\left(l_{1}, l_{2}, l_{3} ; l_{4}, l_{5}, l_{6}\right)$ (that is to be transformed) with respect to the absolute polarity of Euclidean geometry, i.e., $L_{1}=\left(0,0,0 ; l_{1}, l_{2}, l_{3}\right)$. With (2) we find the coordinate representation of this particular quadratic line geometric conchoid transformation as

$$
q:\left(\begin{array}{c}
l_{1} \\
l_{2} \\
l_{3} \\
l_{4} \\
l_{5} \\
l_{6}
\end{array}\right) \mapsto\left(\begin{array}{c}
\delta(\delta-1)\left(l_{1}^{2}+l_{2}^{2}+l_{3}^{2}\right)+\delta l_{1}^{2} \\
\delta l_{1} l_{3} \\
\delta l_{2} l_{3} \\
(1-\delta) l_{1} l_{6}+\delta l_{3} l_{4} \\
(1-\delta) l_{2} l_{6}+\delta l_{3} l_{5} \\
l_{3} l_{6}
\end{array}\right) .
$$

The mapping $q$ is degenerate on the field of lines in the ideal plane, i.e., $q(L)=\mathbf{o}$ for all lines $L=(0,0,0 ; u, v, w)$ with $(u, v, w) \in \mathbb{R}^{3} \backslash\{\mathbf{o}\}$.
Torsality is, in general, not preserved under quadratic line geometric conchoid transformations. Surprisingly, we can show the following result (for an arbitrarily chosen first focal line $L_{0}$ ) which holds in Euclidean three-space $\mathbb{R}^{3}$ :

Theorem 4 The quadratic line geometric conchoid transformation (8) maps cylinders to cylinders.

Proof. Let $L_{0}=(\mathbf{1}, \overline{\mathbf{I}})$ be the first focal line with constant vectors $\mathbf{l}, \overline{\mathbf{l}} \in \mathbb{R}^{3} \backslash\{\mathbf{o}\}$ satisfying $\langle\mathbf{l}, \overline{\mathbf{l}}\rangle=0$. Further, let

$L_{2}=(\mathbf{v}, \overline{\mathbf{v}}): I \subset \mathbb{R} \rightarrow M_{2}^{4}$

be the Plücker representation of the cylinder where the constant vector $\mathbf{v} \in \mathbb{R}^{3} \backslash\{\mathbf{o}\}$ points into the direction of the cylinder's rulings and $\overline{\mathbf{v}}: I \subset \mathbb{R} \rightarrow \mathbb{R}^{3}$ is not constant. Naturally, $\langle\mathbf{v}, \overline{\mathbf{v}}\rangle=0$ for all $t \in I$. Then, the second focal line is given by $L_{1}=(\mathbf{o}, \mathbf{v})$ and is obviously constant. Since we are dealing with lines in Euclidean three-space, we may assume that both $\mathbf{l}$ and $\mathbf{v}$ are unit vectors, i.e., $\langle\mathbf{v}, \mathbf{v}\rangle=\langle\mathbf{l}, \mathbf{l}\rangle=1$. With $\Omega_{01}=\Omega_{12}=1, \Omega_{02}=\langle\mathbf{l}, \overline{\mathbf{v}}\rangle+\langle\overline{\mathbf{l}}, \mathbf{v}\rangle$, and (2), we find

$L_{\delta}=\delta(\delta-1)\left(\begin{array}{c}\mathbf{l} \\ \overline{\mathbf{l}}\end{array}\right)+(1-\delta) \Omega_{02}\left(\begin{array}{l}\mathbf{o} \\ \mathbf{v}\end{array}\right)+\delta\left(\begin{array}{l}\mathbf{v} \\ \overline{\mathbf{v}}\end{array}\right)$

from which we immediately see that the direction vector

$\mathbf{l}_{\delta}=\delta(\delta-1) \mathbf{l}+\delta \mathbf{v}$

is constant since $\mathbf{l}, \mathbf{v} \in \mathbb{R}^{3}$ are constant. Therefore, $L_{\delta}: I \subset$ $\mathbb{R} \rightarrow M_{2}^{4}$ parametrizes a cylinder.

Remark 5 Since the argument $\mathbf{v}$ is constant, the quadratic line geometric conchoid transformation turns out to be linear in the case of the cylinder. According to Cor. 3, torsal ruled surfaces are mapped to torsal ruled surfaces.

It is also possible to verify Thm. 4 via direct computation in terms of coordinates. Then, it is useful to assume that $\mathbf{v}=(0,0,1)$ and the cylinder is erected on the cross section $\mathbf{q}=\left(q_{1}, q_{2}, 0\right): I \subset \mathbb{R} \rightarrow \mathbb{R}^{3}$ in the plane $\langle\mathbf{x}, \mathbf{v}\rangle=0$. Apparently, the cross section of $L_{\delta}$ can be parametrized by

$\mathbf{q}_{\delta}=\frac{1-\delta}{\delta l_{3}}\left(\begin{array}{r}l_{5} \\ -l_{4} \\ 0\end{array}\right)+\frac{1}{\delta}\left(\begin{array}{c}q_{1} \\ q_{2} \\ 0\end{array}\right)$,

and thus, the cross section $\mathbf{q}$ of $L$ undergoes an equiform transformation with scaling factor $\delta^{-1}$.

Eq. (9) shows that the direction of the rulings changes. Fig. 10 shows a cylinder of revolution (elliptic cylinder) and its quadratic conchoidal image. The horizontal cross sections of the cylinder are circles and are mapped to circles. The direction of the cylinder's rulings are changed and the image cylinder is again an elliptic cylinder, but with circular horizontal cross sections. 


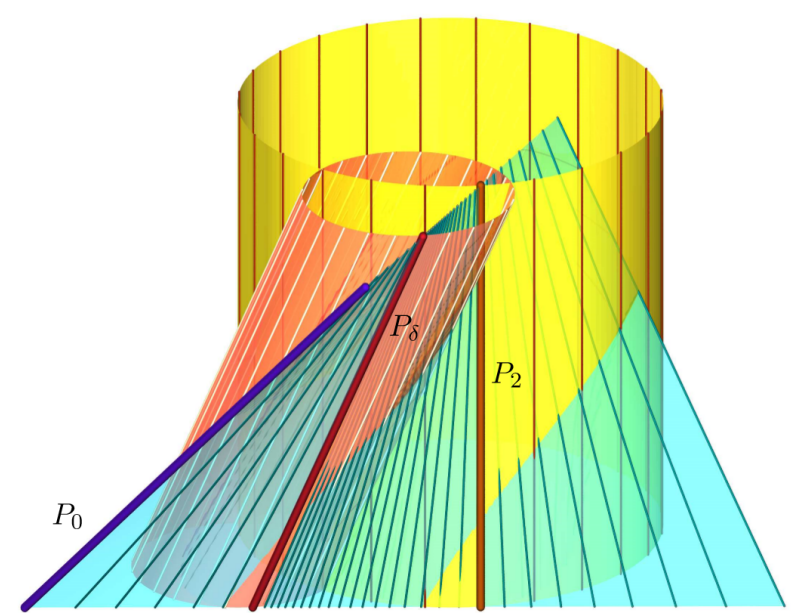

Figure 10: The quadratic line geometric conchoid transform of a cylinder.

\section{Conchoids in sphere geometry}

Many of the results from Sec. 3 dealing with lines can be carried over directly to similar results on spheres. This is mainly based on a mapping that goes back to S. LIE and establishes a one-to-one correspondence between lines and spheres. The mapping is called Lie's line-sphere-mapping which is a projective collineation $\mathbb{P}^{5}(\mathbb{C}) \rightarrow \mathbb{P}^{5}(\mathbb{C})$. Unfortunately, Lie's mapping needs the complex extension of the underlying projective space and mixes up real and complex objects. Therefore, we go a different way and use a coordinatization of the manifold of spheres that was given in [21].

A sphere $S$ in Euclidean three-space $\mathbb{R}^{3}$ can always be given by its equation in terms of Cartesian coordinates as

$$
\begin{aligned}
& S:\left(s_{6}-s_{4}\right)\left(x^{2}+y^{2}+z^{2}\right)- \\
& \quad-2 s_{1} x-2 s_{2} y-2 s_{3} z+\left(s_{6}+s_{4}\right)=0 .
\end{aligned}
$$

For the moment, we assume that $s_{6}-s_{4} \neq 0$. By completing to full squares in the sphere's equation, we find the center

$M=\frac{1}{s_{6}-s_{4}}\left(s_{1}, s_{2}, s_{3}\right)$

and the radius $R$

$R^{2}=\frac{s_{1}^{2}+s_{2}^{2}+s_{3}^{2}+s_{4}^{2}-s_{6}^{2}}{\left(s_{6}-s_{4}\right)^{2}}$.

With the definition

$R=\frac{s_{5}}{s_{6}-s_{4}}$

we find that the six values $s_{i}$ satisfy the homogeneous quadratic equation

$L_{2}^{4}: \Omega_{S}(S, S):=s_{1}^{2}+s_{2}^{2}+s_{3}^{2}+s_{4}^{2}-s_{5}^{2}-s_{6}^{2}=0$.
It is clear that the $s_{i}$ are homogeneous and an interpretation as homogeneous coordinates of points in a projective fivespace is nearby. The coordinates $\mathbf{S}=\left(s_{1}, \ldots, s_{6}\right) \in \mathbb{R}^{6} \backslash\{\mathbf{o}\}$ are called Lie's sphere coordinates. We shall keep in mind that $R$ can be equipped with a sign which can be used to express an orientation of the sphere $S$. The quadric $L_{2}^{4}$ spans $\mathbb{P}^{5}(\mathbb{R})$, is of index one, and therefore, it carries straight lines as maximal subspaces. $L_{2}^{4}$ is called Lie's quadric (cf. $[5,7,20,21])$ and serves as a point model for the set of spheres in Euclidean three-space.

The polar form $\Omega_{S}: \mathbb{R}^{6} \times \mathbb{R}^{6} \rightarrow \mathbb{R}$ describes the polar system of $L_{2}^{4}$. Assume that $S$ and $T$ are two different spheres (non-proportional Lie coordinates) in oriented contact, i.e., the radii (normal vectors) have equal orientation at the point of contact. Then, $S$ and $T$ are conjugate with regard to $L_{2}^{4}$, or equivalently, $\Omega_{S}(S, T)=0$, and vice versa.

The two quadrics $M_{2}^{4}$ and $L_{2}^{4}$ are each others collinear image under Lie's line-sphere-mapping, see [5, 7, 20, 21].

However, the collinear transformation does not map real objects to real ones in general and $L_{2}^{4}$ carries only straight lines, while $M_{2}^{4}$ carries two independent families of planes. Since $L_{2}^{4}$ carries at most straight lines, there exists only one type of regular conics in $L_{2}^{4}$. These conics correspond to one-parameter families of spheres enveloping Dupin cyclides. Hence, the fibers of the sphere geometric conchoid transformation are, loosely speaking, Dupin cyclides (cf. Fig. 11). (More precise, but rather lengthy: The fibers of the sphere geometric conchoid transformation are oneparameter families of spheres enveloping Dupin cyclides.) In analogy to Thm. 2, we can state:

Theorem 5 Let $S_{0}$ and $S_{1}$ be two spheres in Euclidean three-space $\mathbb{R}^{3}$ (not in oriented contact, i.e., $\Omega_{S}\left(S_{0}, S_{1}\right) \neq 0$ ) and let $\delta \in \mathbb{R} \cup\{\infty\}$ be a certain fixed value. Then, the sphere geometric conchoid transformation induces a (regular) automorphic collineation $\lambda$ of $L_{2}^{4}$ that has a fixed line $f$ and a fixed three-space $L$ for its axis. $F$ and $f$ are polar with regard to $L_{2}^{4}$.

Proof. The proof can be kept short. Without loss of generality, we may assume that the focal spheres are given by

$$
\begin{aligned}
& S_{0}=(0,0,0,-1,1,0) \\
& S_{1}=\left(m, 0,0, \frac{1}{2}\left(m^{2}-R^{2}-1\right), R, \frac{1}{2}\left(m^{2}-R^{2}+1\right)\right)
\end{aligned}
$$

are the two focal spheres which are not in oriented contact unless

$(R-1+m)(R-1-m)=0$.

If now $S_{2}=\left(s_{1}, \ldots, s_{6}\right)$ is the sphere to be transformed, then $S_{\delta}$ can be obtained with (2) where $\Omega$ is the polar form with the coordinate matrix $\operatorname{diag}(1,1,1,1,-1,-1)$. 

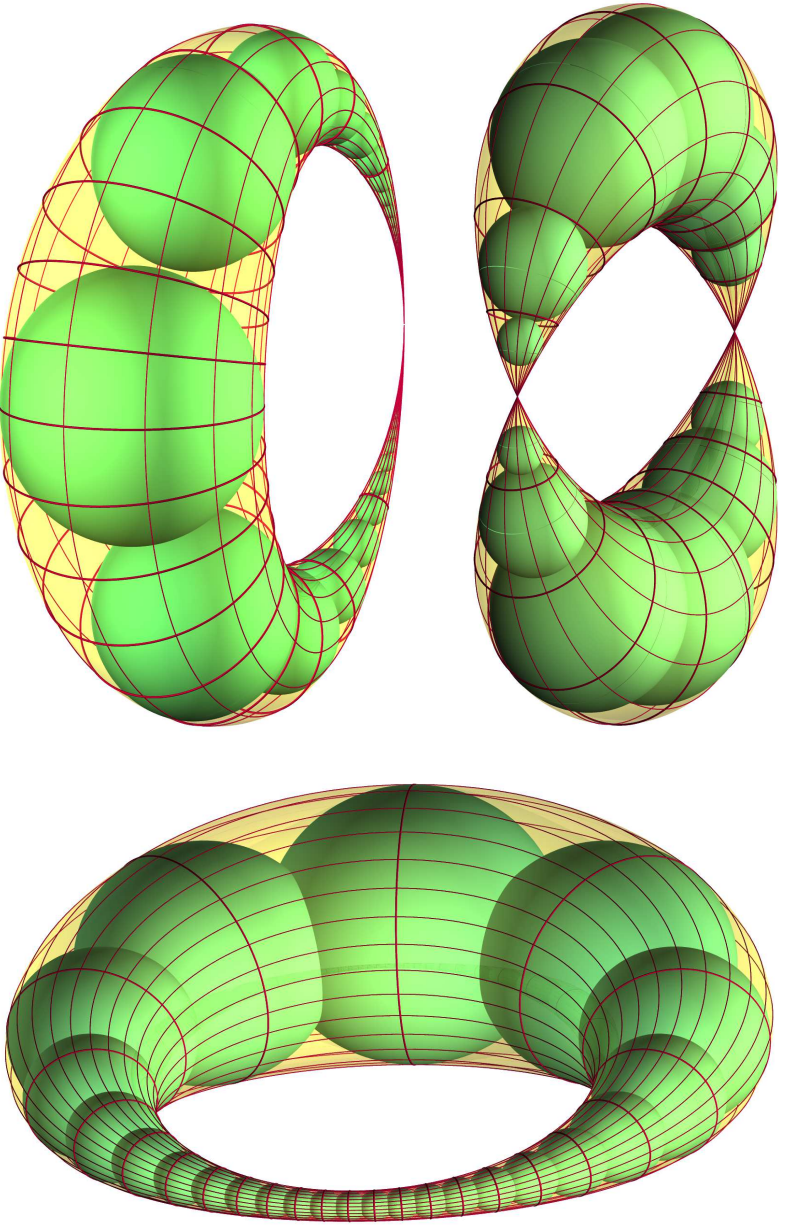

Figure 11: The fibers of the sphere geometric conchoid construction are Dupin cyclides of any type.

In the following, we use the abbreviations

$\rho_{1}=R-m-1, \quad \rho_{2}=R-m+1$.

The coordinate matrix of the linear mapping $S_{2} \mapsto S_{\delta}$ has the three different eigenvalues $t_{1}=\frac{1}{2} \rho_{1} \rho_{2}, t_{2}=\delta^{2} t_{1}$, and $t_{3}=\delta t_{1}$ with the respective algebraic multiplicities $\mu\left(t_{1}\right)=$ $\mu\left(t_{2}\right)=1$ and $\mu\left(t_{3}\right)=4$. Then, it is easily verified that the $\lambda$-invariant subspaces show the same behavior as those belonging to $\kappa$ in the proof of Thm. 2. $F$ and $f$ are polar with regard to $L_{2}^{4}$ according to Cor. 2 .

Remark 6 If the focal spheres $S_{0}$ and $S_{1}$ are in oriented contact, i.e., $\Omega_{S}\left(S_{0}, S_{1}\right)=0, \lambda$ is a projection (singular collineation) onto a one-dimensional subspace of $\mathbb{P}^{5}(\mathbb{R})$, since then, $(R-1+m)(R-1-m)=\rho_{1} \rho_{2}=0$ and the coordinate matrix of $S_{2} \mapsto S_{\delta}$ is of rank 2 .

The above chosen coordinatization of the Euclidean spheres covers more than just Euclidean spheres:
1. (Oriented) Euclidean spheres $S$ are characterized by $s_{6}-s_{4} \neq 0$ (otherwise the quadratic term in (10) would vanish) and $s_{5} \neq 0$, and therefore, $R \neq 0$. Especially, the Euclidean unit sphere $S^{2}$ has Lie coordinates $(0,0,0,1,1,0)$.

2. (Oriented) planes are characterized by $s_{6}-s_{4}=0$. Naturally, the remaining non-vanishing coordinates have to fulfill $s_{1}^{2}+s_{2}^{2}+s_{3}^{2}=s_{5}^{2}$. Sometimes, $s_{5}$ is set to one and $\left(s_{1}, s_{2}, s_{3}\right)$ is then a unit normal vector of the plane

$\varepsilon: 2 s_{1} x+2 s_{2} y+2 s_{3} z-\left(s_{4}+s_{6}\right)=0$.

3. The hyperplane $s_{5}=0$ meets $L_{2}^{4}$ along the regular three-dimensional quadric $s_{1}^{2}+s_{2}^{2}+s_{3}^{2}+s_{4}^{2}-s_{6}^{2}=0$ all of whose points correspond to spheres of radius 0 . However, spheres of radius 0 can be viewed as points $P$ with coordinates

$\mathbf{p}=\left(\frac{s_{1}}{s_{6}-s_{4}}, \frac{s_{2}}{s_{6}-s_{4}}, \frac{s_{3}}{s_{6}-s_{4}}\right)$

but should rather be considered as isotropic cones $\Gamma_{P}$ of Euclidean geometry with the equation

$\Gamma_{P}:\langle\mathbf{x}-\mathbf{p}, \mathbf{x}-\mathbf{p}\rangle=0$.

With $\Gamma_{\mathbf{o}}$ we denote the isotropic cone with the equation $\langle\mathbf{x}, \mathbf{x}\rangle=0$ emanating from the origin $\mathbf{o}=$ $(0,0,0)$ of the coordinate system.

4. Finally, the Lie coordinate vector $(0,0,0,1,0,1)$ turns (10) into a false statement, although it describes a point on $L_{2}^{4}$. It is useful to perform the conformal closure by setting

$U=(0,0,0,1,0,1)$.

Thus, there are four principal types of elements in Lie geometry and any pair out of these four gives rise to a certain sphere geometric conchoid transformation when used as pair of focal spheres. Depending on the nature of the pairs $\left(S_{0}, S_{1}\right)$ of focal spheres, the sphere geometric conchoid transformations turn out to be well-known transformations from specific subgroups of the huge group of contact transformations. We are able show the following:

Theorem 6 1. The sphere geometric conchoid transformation with $S_{0}=U$ and $S_{1}=\Gamma_{\mathbf{0}}$ is an equiform transformation, more precisely a similarity with scaling factor $\delta$.

2. The sphere geometric conchoid transformation with $S_{0}=U$ and $S_{1}=\mathrm{S}^{2}$ is a Laguerre transformation.

3. The sphere geometric conchoid transformation with $S_{0}=\Gamma_{\mathbf{o}}$ and $S_{1}=\mathrm{S}^{2}$ is an inversion. 


\section{Proof.}

1. It means no restriction to assume that the isotropic cone is centered at the origin of the Cartesian coordinate system. Hence,

$U=(0,0,0,1,0,1), \quad \Gamma_{\mathbf{o}}=(0,0,0,1,0,-1)$.

Inserting $S_{0}=U, S_{1}=\Gamma_{\mathbf{o}}$, and $S_{2}=\left(s_{1}, \ldots, s_{6}\right)$ into (2), we find the induced linear mapping

$$
\begin{aligned}
\left(s_{1}, \ldots, s_{6}\right) \mapsto & \left(2 \delta s_{1}, 2 \delta s_{2}, 2 \delta s_{3},\left(1+\delta^{2}\right) s_{4}+\left(\delta^{2}-1\right) s_{6},\right. \\
& \left.2 \delta s_{5},\left(\delta^{2}-1\right) s_{4}+\left(1+\delta^{2}\right) s_{6}\right) .
\end{aligned}
$$

Consequently, spheres with center $\mathbf{m}$ and radius $\rho$ are mapped to spheres with center $\delta \mathbf{m}$ and radius $\delta \rho$, while planes are mapped to planes. Further, points are mapped to points. Fig. 12 shows the action of this mapping.

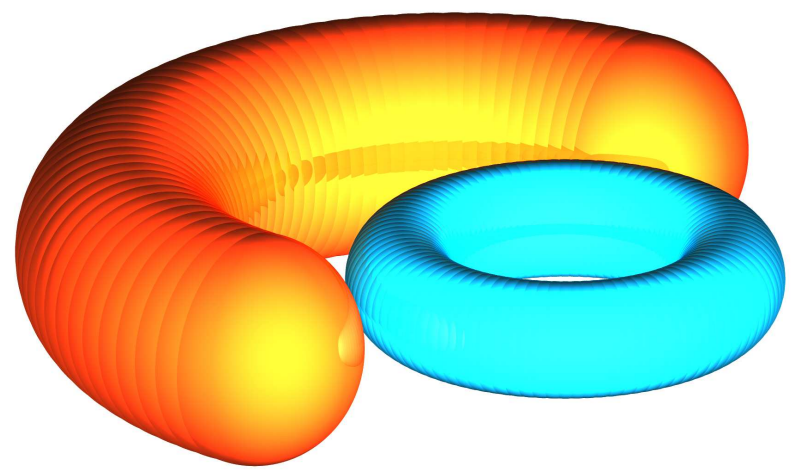

Figure 12: The action of an equiform transformation on one of the one-parameter families of spheres enveloping a torus.

2. In this case, we have $S_{0}=U$ (like in the previous case) and

$S_{1}=\mathrm{S}^{2}=(0,0,0,1,1,0)$.

With $S_{2}=\left(s_{1}, \ldots, s_{6}\right)$ and (2), we find the induced linear mapping

$$
\begin{aligned}
\left(s_{1}, \ldots, s_{6}\right) \mapsto & \left(\delta s_{1}, \delta s_{2}, \delta s_{3}, \delta(\delta-1)\left(s_{4}-s_{5}\right)+\right. \\
& +(\delta-1) s_{6}+s_{4}, \delta s_{5}+(\delta-1)\left(s_{6}-s_{4}\right), \\
& \left.\delta(\delta-1)\left(s_{4}-s_{5}\right)+\delta s_{6}\right) .
\end{aligned}
$$

Obviously, a sphere with center $\mathbf{m}$ and radius $\rho$ is mapped to a sphere with center $\delta \mathbf{m}$ and radius

$$
\frac{\delta s_{5}+(\delta-1)\left(s_{6}-s_{4}\right)}{s_{6}-s_{4}}=\delta \rho+\delta-1 .
$$

Further, a plane with the equation $s_{1} x+s_{2} y+s_{3} z+$ $s_{4}=0$ with $s_{1}^{2}+s_{2}^{2}+s_{3}^{2}=s_{5}^{2}=1$ is mapped to the plane $s_{1} x+s_{2} y+s_{3} z+s_{4}-1+\delta+\delta \rho=0$ which makes the present sphere geometric conchoid a Laguerre transformation, cf. [3, 5, 7]. Fig. 13 shows how the spheres in a torus change under a Laguerre transformation.

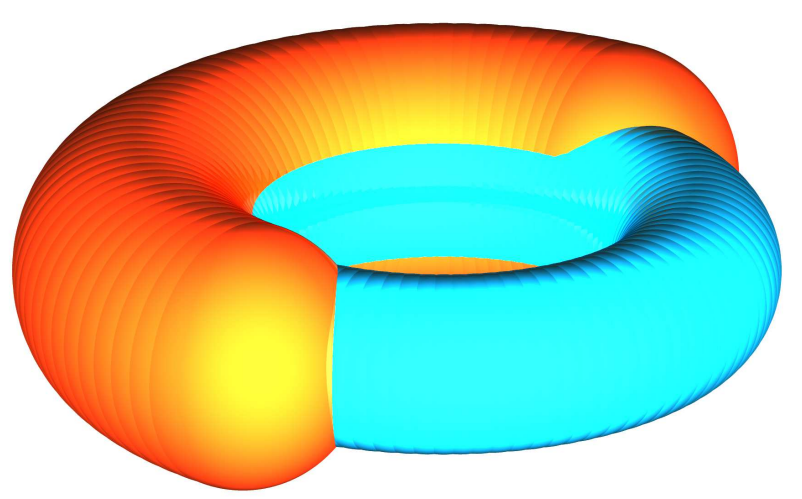

Figure 13: A Laguerre transformation is applied to one of the one-parameter families of spheres enveloping a torus.

3. Finally, we choose the focal spheres

$$
\begin{aligned}
& S_{0}=\Gamma_{\mathbf{o}}=(0,0,0,1,0,-1), \\
& S_{1}=S^{2}=(0,0,0,1,1,0) .
\end{aligned}
$$

Thus, (2) yields the linear mapping

$$
\begin{aligned}
\left(s_{1}, \ldots, s_{6}\right) \mapsto & \left(\delta s_{1}, \delta s_{2}, \delta s_{3}, \delta(\delta-1)\left(s_{4}-s_{5}\right)+\right. \\
& +(1-\delta) s_{6}+s_{4}, \delta s_{5}+(1-\delta)\left(s_{4}+s_{6}\right) \\
& \left.\delta(1-\delta)\left(s_{4}-s_{5}\right)+\delta s_{6}\right)
\end{aligned}
$$

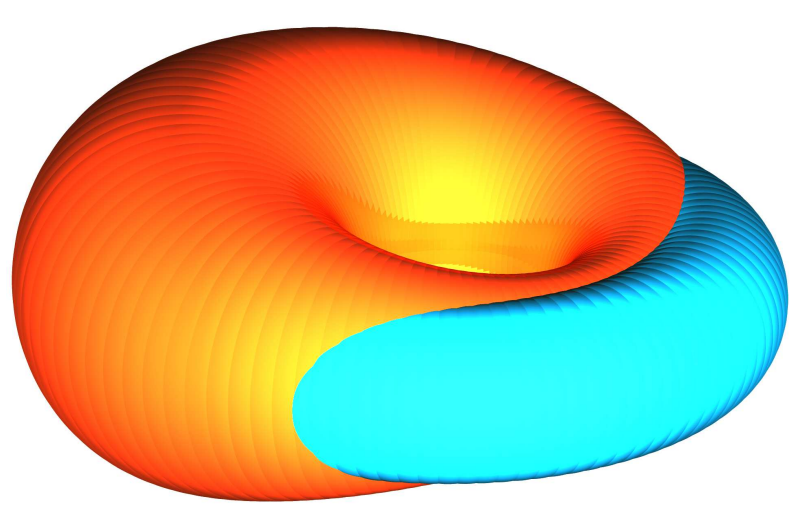

Figure 14: An inversion maps a one-parameter family of spheres enveloping a torus to a one-parameter family of spheres enveloping a Dupin cyclide. 
which maps spheres with center $\mathbf{m}$ and radius $\rho$ to spheres with center

$\frac{\delta \mathbf{m}}{\delta(2 \rho \delta-2 \rho+\delta)+\left(\rho^{2}-\langle\mathbf{m}, \mathbf{m}\rangle\right)(\delta-1)^{2}}$

and radius

$$
\frac{\left(\langle\mathbf{m}, \mathbf{m}\rangle-\rho^{2}\right)(\boldsymbol{\delta}-1)-\rho \delta}{\left(\langle\mathbf{m}, \mathbf{m}\rangle-\rho^{2}\right)(\delta-1)^{2}-\delta(2 \rho \delta-2 \rho+\delta)} .
$$

Obviously, this is an inversion as illustrated in Fig. 14.

\subsection{Quadratic mappings}

Similar to the case of line geometric conchoid transformations, quadratic sphere geometric conchoid transformations can be defined. Therefore, it is only necessary to let the focal sphere $S_{1}$ be a linear image of the sphere $S_{2}$ to be transformed. A linear image of $S_{2}$ means a linear image of the Lie coordinate vector of the sphere $S_{2}$.

We shall have a look at two special types:

Theorem 7 1. The quadratic sphere geometric conchoid transformation with the first focal sphere $S_{0}=$ $\Gamma_{\mathbf{0}}$ and the second focal sphere $S_{1}$ being the polar plane of $(0,0,0)$ with respect to $S_{2}=\left(s_{1}, \ldots, s_{6}\right)$ is a central similarity with center $(0,0,0)$ and scaling factor $\frac{1}{2}(1-2 \delta) \delta^{-2}$, provided that $\delta \neq 0, \frac{1}{2}$.

2. The quadratic sphere geometric conchoid transformation with the first focal sphere $S_{0}=S^{2}=$ $(0,0,0,-1,1,0)$ and the second focal sphere $S_{1}$ being the radical plane of $S_{0}$ and $S_{2}=\left(s_{1}, \ldots, s_{6}\right)$ is a cubic transformation, provided that $\delta \neq 0,1$.

\section{Proof.}

1. The Lie coordinates of the focal spheres are

$S_{0}=(0,0,0,-1,0,1)$,

$S_{1}=\left(s_{1}, s_{2}, s_{3},-\frac{1}{2}\left(s_{4}+s_{6}\right), s_{5},-\frac{1}{2}\left(s_{4}+s_{6}\right)\right)$

with $s_{5}^{2}=s_{1}^{2}+s_{2}^{2}+s_{3}^{2}$. Then, (2) yields

$S_{\delta}=\left((1-2 \delta) s_{1},(1-2 \delta) s_{2},(1-2 \delta) s_{3}\right.$,

$$
\begin{aligned}
& -\frac{1}{2}\left(\delta^{2}\left(s_{4}-s_{6}\right)+s_{4}+s_{6}\right),(1-2 \delta) s_{5}, \\
& \left.\frac{1}{2}\left(\delta^{2}\left(s_{6}-s_{4}\right)-s_{4}-s_{6}\right)\right)
\end{aligned}
$$

where $s_{4}+s_{6} \neq 0$ is canceled, since $\Omega_{01}=2 \Omega_{02}=$ $\Omega_{12}\left(s_{6}-s_{4}\right)^{-1}$. This can be expressed by means of the original sphere data of $S_{2}$ (center $\mathbf{m}=$ $\left(m_{1}, m_{2}, m_{3}\right)$ and radius $\left.\rho\right)$ via

$s_{1}=m_{1}, s_{2}=m_{2}, s_{3}=m_{3}, s_{5}=\rho$,

$s_{4}=\frac{1}{2}\left(\langle\mathbf{m}, \mathbf{m}\rangle-\rho^{2}-1\right), s_{6}=\frac{1}{2}\left(\langle\mathbf{m}, \mathbf{m}\rangle-\rho^{2}+1\right)$ and gives the center $\mathbf{m}_{\delta}$ and radius $\rho_{\delta}$ of the image spheres:

$$
\mathbf{m}_{\delta}=\frac{1-2 \delta}{2 \delta^{2}} \mathbf{m}, \quad \rho_{\delta}=\frac{1-2 \delta}{2 \delta^{2}} \rho .
$$

2. Inserting the Lie coordinates of the two focal spheres

$S_{0}=(0,0,0,-1,1,0)$,

$S_{1}=\left(s_{1}, s_{2}, s_{3}, \frac{1}{2}\left(s_{6}-s_{4}\right), s_{5}, \frac{1}{2}\left(s_{6}-s_{4}\right)\right)$.

into (2) yields

$$
\begin{aligned}
S_{\delta}= & \left(s_{1}\left((1-2 \delta) s_{4}-s_{6}\right), s_{2}\left((1-2 \delta) s_{4}-s_{6}\right),\right. \\
& s_{3}\left((1-2 \delta) s_{4}-s_{6}\right), \delta\left((\delta-1) s_{4}-s_{5}\right)\left(s_{4}-s_{6}\right)- \\
& -\left(s_{4}+s_{5}\right) s_{6}, s_{5}\left((1-2 \delta) s_{4}-s_{6}\right), \\
& \delta\left((1-\delta) s_{4}+s_{6}\right)\left(s_{4}-s_{6}\right)-\left(s_{4}-s_{5}\right) s_{5},
\end{aligned}
$$

which can be reshaped with (14), and finally, (11) and (12) allow us to compute the center and the radius as

$$
\begin{aligned}
& \mathbf{m}_{\delta}=\frac{1}{\delta^{2}}\left((1-\delta)\left(\langle\mathbf{m}, \mathbf{m}\rangle-\rho^{2}\right)+\delta\right) \mathbf{m} \\
& \rho_{\delta}=\frac{1}{\delta^{2}}\left((1-\delta)\left(\langle\mathbf{m}, \mathbf{m}\rangle-\rho^{2}\right)+\delta\right) \rho .
\end{aligned}
$$

Obviously, $\mathbf{m}_{\delta}$ is cubic in the coordinates of $\mathbf{m}$ and $\rho_{\delta}$ is cubic in $\rho$.

\section{Conclusion}

The projective models of various geometries allow us to generalize the well-known conchoid transformation as long as a quadric model exists and a cross ratio can be defined. So far we haven't dealt with singular quadrics such as Blaschke's cylinder model for isotropic geometries. The subspaces contained in singular quadrics may cause problems for the generalized conchoid construction.

The sphere model of Möbius geometry could also be a playground for generalized conchoid constructions. Nevertheless, cross ratios of four complex numbers can also be defined and give rise to a generalized conchoid construction in the Gauss plane.

Finally, there is one special quadric serving as a point model for the set of Euclidean motions: It is Study's quadric $S_{2}^{6} \subset \mathbb{P}^{6}(\mathbb{R})$. Conchoid transformations within Study's quadric may generate special Euclidean motions in Euclidean three space.

\section{Acknowledgement}

I have to express my sincere thanks to a careful reviewer whose suggestions helped to improve the paper. 


\section{References}

[1] A. Albano, M. Roggero, Conchoidal transform of two plane curves, Appl. Algebra Eng. Comm. Comp. 21(4) (2010), 309-328.

[2] W. BENZ, Vorlesungen über Geometrie der Algebren. Springer-Verlag, Berlin-Heidelberg-New York, 1973.

[3] W. BlaschKe, Vorlesungen über Differentialgeometrie III. Springer-Verlag, Berlin, 1929.

[4] E. Brieskorn, H. KNÖRRer, Plane Algebraic Curves. Birkhäuser, Basel - Boston - Stuttgart, 2012.

[5] T.E. CECIL, Lie sphere geometry, Springer, New York; 2nd ed., 2008.

[6] K. FLADT, Analytische Geometrie spezieller ebener Kurven, Akademische Verlagsgesellschaft, Frankfurt am Main, 1962.

[7] O. GIERING, Vorlesungen über höhere Geometrie. Vieweg, Braunschweig - Wiesbaden, 1982.

[8] M. Hamann, B. Odehnal, Conchoidal ruled surfaces, Proc. $15^{\text {th }}$ Int. Conf. Geometry \& Graphics, Aug. 1-5, 2012, Montreal, article No. 089.

[9] J. HoscheK, Liniengeometrie, Bibliographisches Institut, Zürich, 1971.

[10] J.D. LAWRENCE, A catalog of special plane curves, Dover Publications, New York, 1972.

[11] G. LORIA, Spezielle algebraische und transzendente ebene Kurven, B.G. Teubner, Leipzig, 1908.

[12] B. Odehnal, Conchoids on the Sphere, KoG 17 (2014), 43-52.

[13] M. Peternell, D. Gruber, J. Sendra, Conchoid surfaces of rational ruled surfaces, Comp. Aided Geom. Design 28 (2011), 427-435.
[14] M. Peternell, D. Gruber, J. Sendra, Conchoid surfaces of spheres, Comp. Aided Geom. Design 30 (2013), 35-44.

[15] M. Peternell, L. Gotthart, J. Sendra, J.R. SENDrA, Offsets, Conchoids and Pedal Surfaces, $J$. Geom. 106 (2015), 321-339.

[16] J.G. Semple, Algebraic Curves, Clarendon Press, Oxford, 1959.

[17] J.R. SEndRA, J. SEndRA, An algebraic analysis of conchoids to algebraic curves, Appl. Algebra Eng. Comm. Comp. 19(5) (2008), 285-305.

[18] J. SEndRA, J.R. SENDRA, Rational parametrization of conchoids to algebraic curves, Appl. Algebra Eng. Comm. Comp. 21(4) (2010), 413-428.

[19] R. SAUER, Projektive Liniengeometrie, W. de Gruyter \& Co., Berlin und Leipzig, 1937.

[20] E.A. Weiss, Einführung in die Liniengeometrie und Kinematik. B.G. Teubner, Leipzig und Berlin, 1935.

[21] E.A. WEISS, Die geschichtliche Entwicklung der Lehre von der Geraden-Kugel-Transformation VII, Deutsche Math. 3 (1938), 11-35.

[22] H. WiEleitner, Spezielle ebene Kurven, G.J. Göschen'sche Verlagshandlung, Leipzig, 1908.

[23] K. ZINDLER, Liniengeometrie mit Anwendungen I \& II, G.J. Göschen'sche Verlagshandlung, Leipzig, 1902.

\section{Boris Odehnal}

orcid.org/0000-0002-7265-5132

e-mail: boris.odehnal@uni-ak.ac.at

University of Applied Arts Vienna

Oskar-Kokoschka-Platz 2, A-1100 Vienna, Austria 